University of Wollongong

Research Online

Faculty of Engineering - Papers (Archive)

Faculty of Engineering and Information

Sciences

$1-1-2010$

\title{
The ventilated filling box containing a vertically distributed source of buoyancy
}

Paul Cooper

University of Wollongong, pcooper@uow.edu.au

Gary R Hunt

Imperial College London

Follow this and additional works at: https://ro.uow.edu.au/engpapers

Part of the Engineering Commons

https://ro.uow.edu.au/engpapers/3240

\section{Recommended Citation}

Cooper, Paul and Hunt, Gary R: The ventilated filling box containing a vertically distributed source of buoyancy 2010, 39-58.

https://ro.uow.edu.au/engpapers/3240

Research Online is the open access institutional repository for the University of Wollongong. For further information contact the UOW Library: research-pubs@uow.edu.au 


\title{
The ventilated filling box containing a vertically distributed source of buoyancy
}

\author{
PAUL COOPER ${ }^{1} \dagger$ AND GARY R. HUNT ${ }^{2}$ \\ ${ }^{1}$ School of Mechanical, Materials and Mechatronic Engineering, University of Wollongong, \\ Wollongong, NSW 2522, Australia \\ ${ }^{2}$ Department of Civil and Environmental Engineering, Imperial College London, \\ Imperial College Road, London SW7 2AZ, UK
}

(Received 27 January 2009; revised 3 October 2009; accepted 13 October 2009; first published online 10 February 2010)

This paper describes the fluid mechanics within a box containing a vertical plane distributed source of buoyancy. A theoretical analysis is presented that models the development of plumes from such sources in an unconfined ambient of uniform density. Two extensions are considered. The first concerns a sealed box and the second involves the more general situation where the box is ventilated by openings at top and bottom. In the sealed box the stratification develops in much the same way as for a 'filling box' containing a single-point source of buoyancy on the floor. An initial front descends from the ceiling of the box and an asymptotic stratification eventually develops which is continuous in the vertical direction. In the case of the ventilated box it is found that a complex stratification develops where one or more horizontal intrusions are formed by detachment of the plume/boundary layer from the vertically distributed source where the buoyancy of the plume is less than, or equal to, that of the stratified ambient at a given height. Experimental results are presented to demonstrate the validity of the theory. The findings are relevant to both forced and naturally ventilated buildings containing non-adiabatic vertical surfaces.

\section{Introduction}

The 'filling box' problem has received considerable attention in the past because of its relevance to many industrial, domestic and geophysical fluid flows. The problem is to determine the time-dependent stratification resulting from a continuous supply of buoyancy in an enclosure. Baines \& Turner (1969) were the first to carry out a fundamental investigation of the time-dependent stratification resulting from a point source of buoyancy located on the floor of a sealed enclosure. They developed a mathematical model based on the plume theory presented by Morton, Taylor \& Turner (1956) which was extended by others including Worster \& Huppert (1983) who obtained an analytic solution for the transient development of the density field using a linear approximation to the local buoyancy flux in the plume. Germeles (1975) developed a numerical procedure to model the mixing of liquids during the filling of tanks by forced plumes and, more recently, Wells, Griffiths \& Turner

$\dagger$ Email address for correspondence: pcooper@uow.edu.au 

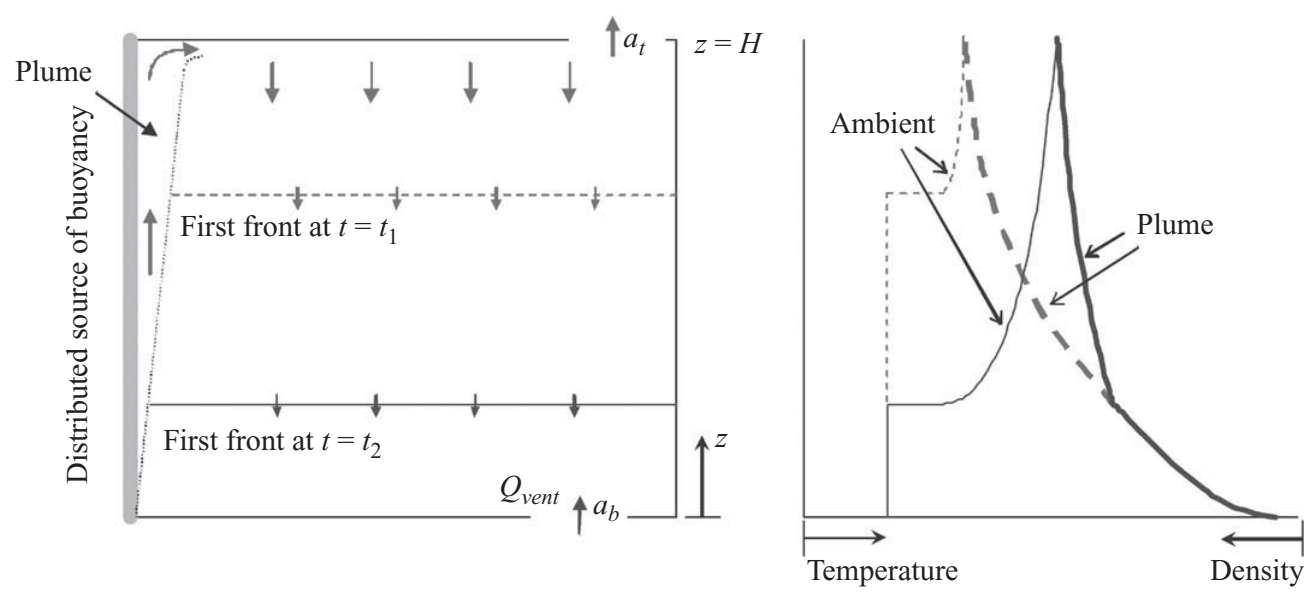

FiguRE 1. Schematic of $(a)$ development of a plume from a plane vertical distributed source of buoyancy in a box with top and bottom vents of areas, $a_{t}$ and $a_{b}$, respectively; $(b)$ position of the first density front at times $t_{1}$ and $t_{2}\left(t_{2}>t_{1}\right)$ and the corresponding density profiles in the plume and the ambient fluid (not to scale).

(1999) investigated the situation where the stratification within a closed space is generated by competing localized and horizontally distributed sources of buoyancy. The steady-state case of an enclosure containing a single-point source of buoyancy was investigated by Linden, Lane-Serff \& Smeed (1990). Subsequently, their work was extended to cover two-point sources (Cooper \& Linden 1996) and multiple-point sources (Linden \& Cooper 1996).

The present work deals with the stratification that develops in a confined space from a vertically distributed source of buoyancy, as shown schematically in figure 1, where a rising boundary layer, or plume, develops adjacent to the source. The primary motivation for our research arises from the need to understand the air flow in naturally and mechanically ventilated buildings and to predict the thermal stratification in ventilated spaces which contain vertical surfaces that are heated or cooled. This is a research topic that has considerable practical significance (Linden 1999), particularly, as building designers and architects have not previously had a means of estimating thermal stratification in these situations. Practical applications include rooms with vertical surfaces heated by incident solar radiation or with surfaces cooled by heat transmission to adjacent spaces or the exterior.

The vertically distributed source of figure 1 leads to a displacement ventilation flow which is similar in nature to that generated by a point source of buoyancy in a sealed or a ventilated box. However, one of the key features of a vertically distributed source in a ventilated box is that the nature of the density field that develops within the box means that it is possible for the boundary layer or plume adjacent to the source to detach from the source and to form one or more horizontal intrusions into the local ambient where the plume becomes less buoyant than the local ambient fluid as it rises. Above this point of detrainment a new plume forms due to the injection of buoyancy from the source over the entire height of the enclosure. A similar situation is observed when a bubble plume rises through a stably stratified environment as discussed by McDougall (1978). If the ambient stratification is sufficiently strong then the fluid entrained into the bubble plume may cease to rise at a given height and will form an intrusion into the ambient. However, the column of bubbles will continue to rise and a new plume forms immediately above the intrusion. This behaviour is not possible in the case of a plume from a point source of buoyancy, which will simply 
cease to rise when it reaches the height of neutral buoyancy and form a horizontal intrusion as described by Morton et al. (1956).

The steady-state density field established by a vertically distributed source in a naturally ventilated enclosure was modelled by Linden et al. (1990). They assumed the plume, or boundary layer, adjacent to the vertical source to be fully turbulent, and postulated that the requirements for conservation of volume and buoyancy fluxes must lead to the development of a stable steady-state stratification comprising a number of fully mixed layers of equal depth $h$. The plume was assumed to detrain and form intrusions at the density interfaces between the fully mixed layers and to start anew at each of these levels. However, the saline solution experiments of Linden et al. (1990) showed only weak evidence of this layering as did the experiments of Chen, Li \& Mahoney (2001) in their study of a vertically distributed source of bubbles in a naturally ventilated enclosure. Sandberg \& Lindstrom (1987) used resistance heating in air to model the flow produced by a heated vertical cylinder extending from floor to ceiling in a non-insulated model. They observed thermal boundary layers on the sidewalls of the enclosure and also detrainment from different levels of the distributed line plume which gave rise to an almost linear variation in temperature in the upper layer. In the present work, we have taken a more comprehensive and rigorous approach to both the theoretical and experimental modelling of the situation first considered by Linden et al. (1990).

\section{The theoretical model}

The geometry of the problem is shown schematically in figure 1 where a plane vertically distributed source of buoyancy of width $L$ is located on one wall of a box of height $H$ and uniform horizontal cross-sectional area $A$. The source emits a buoyancy flux $\Phi$ per unit area and zero fluxes of volume and momentum. A plane turbulent boundary layer, or plume, develops on the wall and forms a buoyant layer at the top of the box. The box may be sealed, mechanically ventilated with a fixed ventilation flow or naturally ventilated. We first consider how the plume from such a buoyancy source develops in an unconfined environment.

\subsection{The plume/boundary layer equations}

Here we adopt an approach to the modelling of the two-dimensional plume which is similar to that of Morton et al. (1956) for point sources of buoyancy. Effects of the source on the development of the boundary layer, other than those directly associated with the supply of buoyancy, are neglected. In particular, viscous effects in the boundary layer adjacent to the vertical surface and the height of the laminar portion of the boundary layer near the leading edge (at $z=0$ ) are assumed to be negligible. In a fully turbulent natural convection boundary layer, the effect of the presence of the wall on the bulk properties of the plume is very minor. From the experimental results of Vliet \& Liu (1969) on velocity profiles in turbulent natural convection boundary layers adjacent to uniformly heated plates, it can be seen that the region adjacent to the wall where the velocity is appreciably reduced by viscous effects accounts for less than $0.25 \%$ of the total volumetric flow in the boundary layer. Moreover, for fully turbulent natural convection boundary layers the Grashof number, a measure of the ratio of buoyancy forces to viscous forces, is very large $\left(>10^{9}\right)$. For such situations the assumption that the influence of viscous forces is small is therefore appropriate and from here onward we refer to the boundary layer as a plume. We also assume that the entrainment assumption is valid, i.e. the ratio 
between the horizontal velocity of fluid entrained into the plume and the mean vertical velocity $w$ in the plume is constant, and we denote this entrainment constant as $\alpha$.

Thus, the equations representing the conservation of the fluxes of volume, momentum and buoyancy in the plume per unit horizontal width of source are as given below. These incorporate the usual Boussinesq and entrainment assumptions (Linden 1999) and make use of 'top-hat' profiles to represent the plume vertical velocity and buoyancy with respect to the local ambient, the latter denoted by $\Delta=g\left(\rho_{e}-\rho\right) / \rho_{1}$, where $\rho(z)$ is the density of the plume, $\rho_{e}(z)$ is the density of the ambient fluid and $\rho_{1}$ is a reference density:

$$
\frac{\mathrm{d}(b w)}{\mathrm{d} z}=\alpha w, \quad \frac{\mathrm{d}\left(b w^{2}\right)}{\mathrm{d} z}=b \Delta-\varepsilon \quad \text { and } \quad \frac{\mathrm{d}(b w \Delta)}{\mathrm{d} z}=b w \frac{\partial \Delta_{e}}{\partial z}+\Phi,
$$

where $b$ is the width of the plume, $\Delta_{e}=g\left(\rho_{e}-\rho_{1}\right) / \rho_{1}$ is the buoyancy of the ambient fluid and $\varepsilon$ represents the influence of wall shear stress, which is assumed to be negligible in the situation at hand, as discussed above. For convenience, (2.1) may be rewritten in terms of the volume flux, $Q=b w$, momentum flux, $M=b w^{2}$ and buoyancy flux, $F=b w \Delta$, per unit width of the plume as follows:

$$
\frac{\mathrm{d} Q}{\mathrm{~d} z}=\alpha \frac{M}{Q}, \quad \frac{\mathrm{d} M}{\mathrm{~d} z}=\frac{Q F}{M} \quad \text { and } \quad \frac{\mathrm{d} F}{\mathrm{~d} z}=Q \frac{\partial \Delta_{e}}{\partial z}+\Phi=-Q N^{2}+\Phi,
$$

where $N$ is the buoyancy frequency of the ambient fluid.

\subsection{Plume in a uniform ambient}

A similarity solution of (2.2) exists in the case of a uniform ambient where $N^{2} \equiv 0$ and therefore $F=z \Phi$. This solution is given by

$$
\left.\begin{array}{rlrl}
Q & =\frac{3}{4}\left(\frac{4}{5}\right)^{1 / 3} \alpha^{2 / 3} \Phi^{1 / 3} z^{4 / 3}, & M & =\frac{3}{4}\left(\frac{4}{5}\right)^{2 / 3} \alpha^{1 / 3} \Phi^{2 / 3} z^{5 / 3}, \\
\Delta & =\frac{4}{3}\left(\frac{5}{4}\right)^{1 / 3} \alpha^{-2 / 3} \Phi^{2 / 3} z^{-1 / 3}, & w & =\left(\frac{4}{5}\right)^{1 / 3} \alpha^{-1 / 3} \Phi^{1 / 3} z^{1 / 3}
\end{array}\right\}
$$

The form of these equations is similar to that for point or line source plumes (e.g. Linden et al. 1990). The volume flux of the vertically distributed source plume, for example, has a somewhat stronger dependency upon elevation $z$ than is the case for the two-dimensional (horizontal) line plume wherein $Q$ is directly proportional to the height above the source. Whereas the volume flux in an axisymmetric point source plume is proportional to $z^{5 / 3}$, for a vertically oriented line source axisymmetric plume it is proportional to $z^{2}$ (Linden et al. 1990).

\subsection{The sealed filling box}

Our analysis follows a similar path to both Baines \& Turner (1969) and Worster \& Huppert (1983). We assume that the flow in the plume is two-dimensional and fully turbulent over the entire height of the buoyancy source (from $z=0$ to $z=H$ ), that the fluid within the box is initially uniform and quiescent and that the horizontal cross-sectional area $A$ of the box is constant with height. In practice, the plume will act as a 'starting' plume immediately after activation at $t=0$, however, for present purposes the plume is assumed to be adequately described by (2.1) for $t>0$. The passive (perfectly insulating) walls of the box are assumed to have no viscous effect on the flow, the effect of the aspect ratio of the box $(H L / A)$ on the flow field is neglected and we assume that the plume continually lays down a thin layer of buoyant fluid at the top of the box, i.e. there is no overturning (Baines \& Turner 1969; 
Kaye \& Hunt 2007). Following Baines \& Turner (1969) and Worster \& Huppert (1983) we assume that molecular diffusion can be neglected and that changes in the ambient stratification are sufficiently slow that flow within the plume may be represented by (2.2), i.e. that the aspect ratio of the box is small and the flushing time scale for the box is much greater than the time for the plume to rise through the box, i.e. $(4 / 3) A /(\alpha L H) \gg 1$ (cf. Bolster \& Caulfield 2008). The development of the buoyancy field is then given by

$$
\frac{\partial \Delta_{e}}{\partial t}=Q \frac{L}{A} \frac{\partial \Delta_{e}}{\partial z}
$$

To arrange the governing differential equations in their simplest non-dimensional form the following variables are defined as:

$$
\left.\begin{array}{l}
\zeta=z H^{-1}, \quad \tau=\alpha^{2 / 3} H^{1 / 3} L A^{-1} \Phi^{1 / 3} t, \quad \delta=\alpha^{2 / 3} H^{1 / 3} \Phi^{-2 / 3} \Delta_{e}, \\
f=H^{-1} \Phi^{-1} F, \quad q=\alpha^{-2 / 3} H^{-4 / 3} \Phi^{-1 / 3} Q, \quad m=\alpha^{-1 / 3} H^{-5 / 3} \Phi^{-2 / 3} M .
\end{array}\right\}
$$

Here the fundamental time scale chosen to characterize the transient development of the flow is the flushing time, i.e. the time for the plume flow at the top of the box (originally containing fluid of uniform density) to displace a volume of fluid equivalent to that in the box. The governing equations for the filling box are then

$$
\frac{\mathrm{d} q}{\mathrm{~d} \zeta}=\frac{m}{q}, \quad \frac{\mathrm{d} m}{\mathrm{~d} \zeta}=\frac{q f}{m}, \quad \frac{\mathrm{d} f}{\mathrm{~d} \zeta}=q \frac{\partial \delta}{\partial \zeta}+1 \quad \text { and } \quad \frac{\partial \delta}{\partial \tau}=q \frac{\partial \delta}{\partial \zeta} .
$$

The buoyancy of the 'first front' of fluid $\delta_{0}$ laid down by the plume at $t=0$ is equal to the buoyancy of the plume arriving at the ceiling of the box. The flow of the plume in the uniform ambient below the first front is therefore given by

$$
f=\zeta, \quad q=\frac{3}{4}\left(\frac{4}{5}\right)^{1 / 3} \zeta^{4 / 3} \text { and } m=\frac{3}{4}\left(\frac{4}{5}\right)^{2 / 3} \zeta^{5 / 3}
$$

It follows that the first front descending through the filling box has a buoyancy given by

$$
-\delta_{0}=\left.\frac{f}{q}\right|_{\zeta=1}=\frac{4}{3}\left(\frac{5}{4}\right)^{1 / 3} \approx 1.436,
$$

and the position of this first front $\zeta_{0}$ is determined from the conservation of volume in the box as a whole such that

$$
\frac{\mathrm{d} \zeta_{0}}{\mathrm{~d} \tau}=-\left.q\right|_{\zeta_{0}}=-\frac{3}{4}\left(\frac{4}{5}\right)^{1 / 3} \zeta_{0}^{4 / 3}
$$

which may be integrated to give $\zeta_{0}=\left[1+\left(\frac{1}{4}\right)\left(\frac{4}{5}\right)^{1 / 3} \tau\right]^{-3}$. In terms of dimensional parameters, the height of the first front above the base of the box is therefore

$$
\frac{z_{0}}{H}=\left[1+\frac{1}{4}\left(\frac{4}{5}\right)^{1 / 3} \alpha^{2 / 3} L A^{-1} H^{1 / 3} \Phi^{1 / 3} t\right]^{-3}
$$

Thus, the time scale associated with the flow is $t^{*} \sim A H^{-1 / 3} \Phi^{-1 / 3} L^{-1}$.

Both Baines \& Turner (1969) and Worster \& Huppert (1983) invoked the assumption that as $\tau \rightarrow \infty$ the additional buoyancy from the source added over a small period of time is distributed uniformly throughout the box. The density of the stratified fluid then changes linearly with time which leads to the condition $\partial \delta / \partial \tau=-1$. However, in the present problem, the form of $f_{\infty}(\zeta)$ is not obvious 

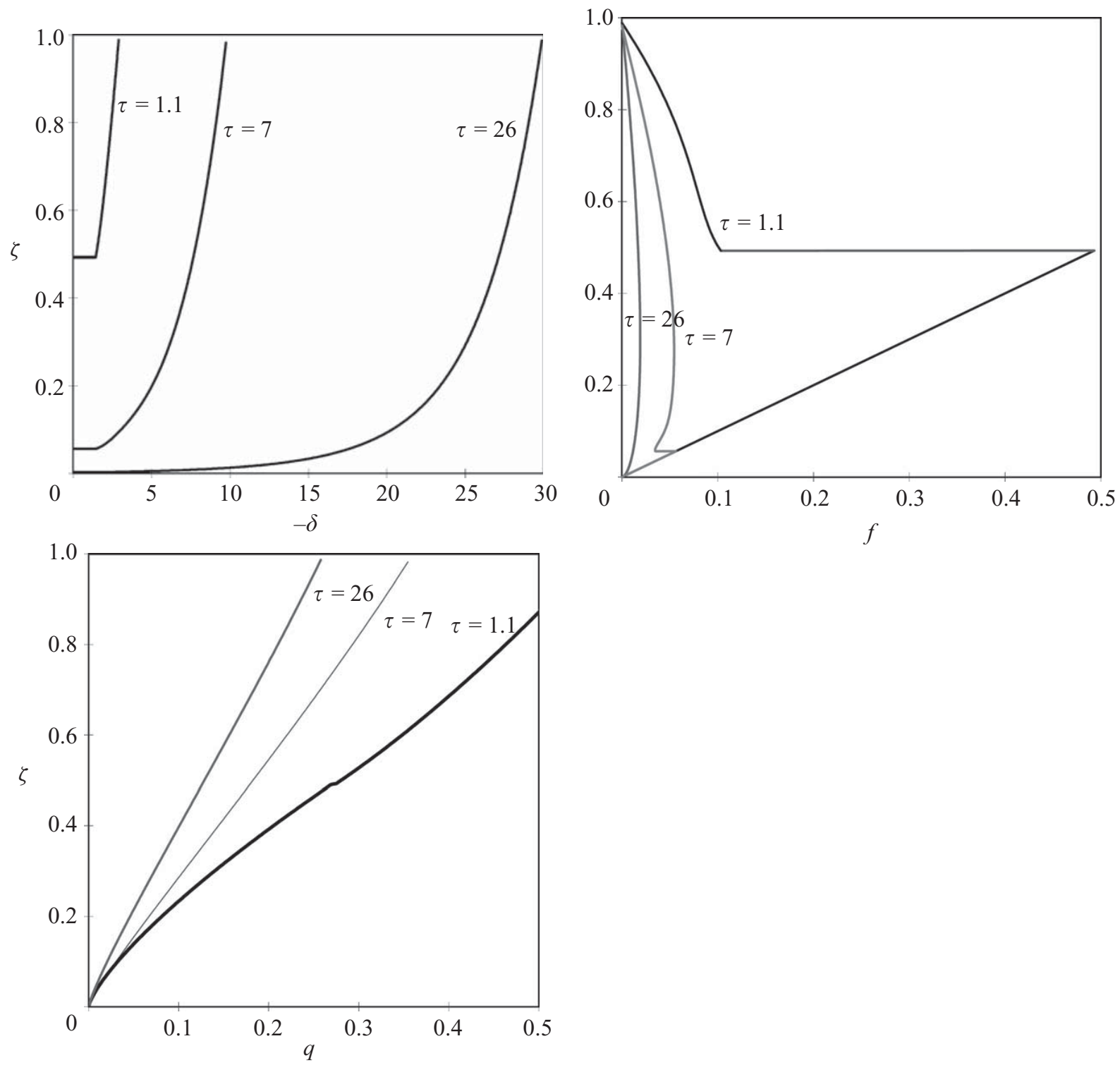

FIGURE 2. Development of the non-dimensional ambient density field $\delta$, local plume buoyancy flux $f$ relative to the ambient and volume flux $q$ in a sealed filling box containing a vertical plane distributed source of buoyancy with increasing time $\tau$.

since the boundary conditions require that $f(1)=f(0)=0$ and the present authors have resorted to a numerical solution of the governing equations. The transient development of the stratification was therefore determined by the solution of (2.6) using a numerical scheme similar to that of Germeles (1975). The density of the ambient fluid in the box $\delta$ as a function of $\zeta$ and $\tau$ was determined by tracking the position of elements leaving the plume at the top of the box where the plume delivers fluid to the ambient. The time step between calculations of plume and ambient fluid velocities and densities was set dynamically to ensure that the maximum vertical change of position of any element in the ambient fluid was held below a prescribed maximum $\left(\Delta \zeta_{\max }<10^{-3}\right)$.

The evolution of the density field shown in figure 2 is similar to that for the filling box with a point source of buoyancy as reported by Baines \& Turner (1969) and Worster \& Huppert (1983). The buoyant layer increases in depth, maintaining the first front buoyancy step $\delta_{0}$. The buoyancy flux in the plume increases linearly with respect to $\zeta$ (figure 2) in the original ambient fluid. At $\zeta=\zeta_{0}$ there is a step change 
in $f$ as the plume passes through the first front. The volume flux in the plume at a given height decreases monotonically with time (figure 2) and the asymptotic state (as $\tau \rightarrow \infty$ ) of the system as a whole is one where vertical velocities tend to zero.

\subsection{The ventilated filling box}

The governing equations of $\S 2.3$ also apply with some modification when the box is ventilated by either natural or forced displacement ventilation as shown schematically in figure 1. In the natural ventilation situation a buoyant layer is formed in the upper part of the box by the distributed source which drives a volume flow rate $Q_{\text {vent }}$ per unit width of source through the upper and lower vents of areas $a_{t}$ and $a_{b}$, respectively. We assume the ventilation flow (e.g. through the base vent) does not affect the dynamics of the rising plume or mix with the developing stratification. We can then use an approach similar to that of Linden et al. (1990) and the magnitude of the natural ventilation flow is determined from the balance between the volume integrated buoyancy of the stratified layer and the pressure losses due to the flow through the vents such that

$$
Q_{v e n t}=\frac{A^{*}}{L}\left(\int_{0}^{H} \Delta_{e} \mathrm{~d} z\right)^{1 / 2} .
$$

The 'effective vent area' $A^{*}$ is defined in Cooper \& Linden (1996) as

$$
A^{*}=2^{1 / 2} c_{d} a_{t} a_{b}\left(\left(c_{d} a_{t}\right)^{2} / c+a_{b}^{2}\right)^{-1 / 2},
$$

where $c$ is the pressure loss coefficient associated with the inflow through a sharpedged opening and $c_{d}$ is a discharge coefficient that accounts for the vena contracta arising at the downstream side of the sharp-edged upper vent. In terms of the dimensionless variables (2.5) the ventilation flow rate is therefore

$$
q_{v e n t}=\frac{A^{*}}{\alpha H L}\left(\int_{0}^{1} \delta \mathrm{d} \zeta\right)^{1 / 2},
$$

and the development of the buoyancy field (cf. (2.6)) is then given by

$$
\frac{\partial \zeta}{\partial \tau}=\left(q-q_{v e n t}\right) \frac{\partial \delta}{\partial \zeta}
$$

This represents an integral constraint on (2.6) where $A^{*} /(\alpha H L)$ is a non-dimensional vent area that determines the stratification within the box and is similar to $A^{*} / H^{2}$ for the case of a ventilated filling box containing a point source of buoyancy (Linden et al. 1990). One major difference between the sealed and ventilated filling boxes is that in the ventilated case a steady-state stratification will eventually be reached, since the buoyancy flux supplied by the source is lost through the top vent.

It is possible under some circumstances for the plume to have a buoyancy less than or equal to that of the local ambient fluid at a given height in the ventilated filling box, as a result of the strongly stratified ambient layer of fluid in the top of the box (as discussed by Linden et al. 1990). This situation may occur under either transient or steady conditions. The plume will then cease to rise as it is forced to 'detrain' from the vertical wall and must spread horizontally to form an intrusion into the ambient somewhat above the neutral buoyancy height, i.e. in a manner similar to that of a plume from a point source rising in a stratified environment, which will reach its neutral buoyancy height and form a horizontal intrusion (Morton et al. 1956). Assuming that the entire plume detrains at this height then a new plume must 
start immediately above the intrusion due to the continuous addition with height of buoyancy flux from the source.

This 'detrainment' of the plume does not occur in the case of a plume from a singlepoint source of buoyancy on the base of a ventilated box. A point source will generate a plume that rises through the ambient fluid until it reaches the interface between the fluid at external ambient density (which has entered via the lower opening of the box) and the buoyant layer, fed by the plume, in the top of the box. Under steady-state conditions the local buoyancy of the plume at the interface is equal to that of the buoyant layer above and hence the plume mixes completely and the homogeneity of the upper layer is maintained. Indeed the fluid from the plume reaches the top of the box by virtue of its vertical momentum at the interface. It is only under transient conditions that the plume from a point source is prevented from rising to the top of the box, an example being when the buoyancy flux of the source is decreased at some point in time after steady state has been reached. The plume will then form a new layer of fluid beneath the buoyant layer in the top of the box (Bolster \& Caulfield 2008). However, in the present situation buoyancy flux is added continuously from the distributed source at all elevations in the box. This leads to the possibility that the buoyancy flux added in upper regions of the box results in a stratification sufficiently intense that the plume will be unable to penetrate the upper layers of ambient fluid and must detrain.

Linden et al. (1990) first considered the case of a vertically distributed source in a ventilated box. Subject to a number of idealizations, they postulated that 'at any level where the volume flux in the plume is not equal to the volume flux out of the box there must be vertical motion exterior to the plume, and for the state to be steady fluid elements exterior to the plume must move along surfaces of constant density (assuming diffusion to be negligible)'. Thus, a finite number of fully mixed layers must develop, with $n$ sharp density interfaces between. Here we apply the model of Linden et al. (1990) to the present case of a plane, vertically distributed source and an analytical solution for the stratification may be found if it is assumed that the depth of the uppermost buoyant layer is the same as the depth $\Delta \zeta$ of the lower layers. The non-dimensional local buoyancy flux $f$ and volume flux $q$ at each interface are then given by (2.7). The buoyancy change $\Delta \delta$ across each interface is

$$
\Delta \delta=\frac{4}{3}\left(\frac{5}{4}\right)^{1 / 3}(n+1)^{1 / 3},
$$

and the relationship between the number of interfaces and the discrete values of non-dimensional vent area for this special case is as follows,

$$
\left(\frac{4}{3}\right)^{3} \frac{5}{4}\left(\frac{A^{*}}{\alpha H L}\right)^{2}=\frac{1}{n(n+1)^{3}} .
$$

\subsection{Numerical model of a ventilated filling box}

The general case of transient development of the stratification in a ventilated box was determined by a numerical solution of (2.6) and (2.4) following a similar methodology to Germeles (1975), either for forced ventilation by prescribing $q_{v e n t}$ or for natural ventilation by assuming a given value of non-dimensional vent area $A^{*} /(\alpha H L)$, and incorporating the integral constraint (2.14). In addition, an algorithm was developed to allow the plume to form one or more horizontal intrusions in any horizontal plane within the box where the local ambient fluid density was equal to or less than that in the plume. In the event of an intrusion forming, the plume volume, momentum 
and buoyancy fluxes in the numerical scheme were set to zero immediately above this point and a new plume started. It was assumed for the purposes of this simulation that an intrusion spread instantaneously throughout the appropriate horizontal plane in the box and that the depth of the intrusion outflow was negligible. The numerical scheme then modelled the increase in depth of the intrusions with time through the conservation of volume throughout the box.

A key finding from our initial work was that the type of multi-layered steady-state stratification postulated by Linden et al. (1990), which comprises a number of fully mixed layers of equal depth, is not physically possible as it is inherently unstable with respect to small perturbations in plume flow or ambient stratification. This arises from the implicit assumption in the theory of Linden et al. (1990) that the buoyancy is uniform across the plume width at a given height and thus all the fluid which forms an intrusion at a point of detrainment must be of that same buoyancy. For example, consider the case where $A^{*} /(\alpha H L)=0.1097$. The multi-layer stratification predicted by (2.15) and (2.16) would result in the plume detraining at $\zeta=0.25$ to form a buoyant layer, of uniform density, from $0.25 \leqslant \zeta \leqslant 0.5$. Should a small increase occur in the local buoyancy of the plume arriving at $\zeta=0.25$ then the plume would pass through the entire (uniform) buoyant layer above and detrain at $\zeta=0.5$ where a new intrusion would form with a buoyancy somewhere between the buoyancy of the layers above and below. This in turn would affect the stratification above this level. Thus, the stratification in the box would be unsteady if the plume is assumed to detrain with a single, i.e. infinitesimally small range of, buoyancy even after significant time has elapsed (e.g. $\tau>100$ ), a fact confirmed by our initial numerical modelling.

In reality, the plume has a non-uniform buoyancy profile with respect to width at a given height. It follows that parcels of fluid from the plume with higher buoyancy would be neutrally buoyant at higher elevations, and thus would detrain at higher elevations. This in turn results in the intrusion having a finite depth. We have therefore developed a more realistic numerical model of the detrainment process whereby the plume detrains with a finite range of buoyancy and forms an intrusion of finite depth in the ambient. Our development of the associated numerical model was informed by previous work on plumes developing in stratified environments from both point and linear sources of buoyancy (e.g. Morton et al. 1956). Of particular relevance is the work of Baines (2002) on the development of a two-dimensional plume adjacent to a vertical wall in a stratified environment. Baines carried out experiments to quantify the non-dimensional depth of penetration of the plume and he developed a theoretical framework for modelling the development of the plume using a non-dimensional buoyancy parameter and the local plume Richardson number to characterize the properties of the plume. Baines' model was verified by his saline solution experiments where the negatively buoyant two-dimensional plume was found to descend to, and then pass through, the depth at which it was neutrally buoyant with respect to the ambient whereupon it 'sprang back' to form a horizontal intrusion of finite thickness. As in the case of Baines' work, the thickness of the intrusions in the present study are, in principle, a function of the local Richardson number, ambient fluid buoyancy frequency, plume width, etc.

For present purposes our requirement was to overcome the unrealistic instability of the model of Linden et al. (1990) and to develop a simple model of a detraining plume such that at the point of detrainment the plume was assumed to have a triangular buoyancy profile centred on the mean plume buoyancy at that elevation. The detraining plume was assumed to form an intrusion with this same buoyancy profile and to enter the ambient fluid over a finite depth with a triangular profile of 
horizontal velocity $u_{\text {int }}$ with respect to local buoyancy (more complex profiles could be utilized if required). Thus, wherever the rising plume became neutrally buoyant with respect to the ambient (of local buoyancy $\delta_{\text {neut }}$ ) then the resulting intrusion was assumed to have a range of buoyancy $\Delta \delta_{\text {int }}$. The difference between the buoyancy of fluid at the top and bottom of this intrusion was taken to be a prescribed small fraction $\lambda_{i n t}$ of the total difference in buoyancy between ambient fluid at the top and bottom of the box, i.e. for all intrusions,

$$
\Delta \delta_{i n t}=\lambda_{i n t}\left(\delta_{\zeta=1}-\delta_{\zeta=0}\right) .
$$

In the numerical model all the fluid from a detraining plume was assumed to enter the ambient by incorporating the above refinement to any Germeles (1975) numerical ambient layers which held fluid within the range of buoyancy of the intrusion $\left(\Delta \delta_{\text {neut }}-\delta_{\text {int }} / 2\right)<\delta<\left(\Delta \delta_{\text {neut }}+\delta_{\text {int }} / 2\right)$. The horizontal detrainment velocity, $u_{\text {int }}$, within the intrusion was taken to be proportional to the difference in local ambient buoyancy and neutral buoyancy, i.e. $u_{\text {int }} \propto\left|\delta-\delta_{\text {neut }}\right| / \delta_{\text {int }}$.

Although this choice of an arbitrary depth, or range of buoyancy, of the intrusion did not capture the complex, and as yet unquantified, physics of the detrainment process, it did serve to overcome the limitations of the model of Linden et al. (1990) and delivered a stable steady-state stratification. Importantly it also ensured that the total buoyancy and volume fluxes entering the stratified ambient from the detraining plume were equal to those of our initial zero-depth intrusion model. This scheme successfully realized our aim of introducing intrusions of finite depth within the Germeles (1975) numerical scheme.

\subsubsection{Forced ventilation}

The implementation of our refined numerical model with finite intrusion thicknesses produced a steady-state stratification which was stable with respect to small perturbations to the ambient fluid stratification or plume flow. The predicted stratification of the ambient was relatively insensitive to the magnitude of the nondimensional thickness of the intrusions for $0.01<\lambda_{i n t}<0.05$. An example of the results from this modified numerical scheme with a finite intrusion thickness is shown in figure 3 where the forced ventilation flow rate was fixed at $q_{v e n t}=0.1097$, which is the flow rate predicted by (2.7) and (2.15) to produce a four-layer stratification with three interfaces. The stratification predicted by our refined numerical scheme with a finite intrusion depth $\left(\lambda_{\text {int }}=0.02\right)$ is compared with the analytical result from (2.15) and (2.16), following Linden et al. (1990), and with the results of our initial numerical model with infinitesimally thin intrusions. It can be seen that for this particular ventilation flow rate the first front is predicted to descend to near the steady-state height of the ambient layer in the bottom of the box $(\zeta=0.25)$ over a period of $\tau \sim 10$. Following a period of readjustment, the plume and ambient reach a steady state, after $\tau \sim 50$, such that there are two points at which the plume detrains, i.e. at the top of the ambient layer $(\zeta=0.25)$ and at $\zeta \approx 0.7$. A relatively smooth change in buoyancy of the ambient over the height of the box, with the exception of the points where the plume detrains, is observed as compared to the sharp interfaces between the fully mixed layers predicted by the model of Linden et al. (1990).

\subsubsection{Natural ventilation}

Results from the numerical model of stratification and plume flow rate in a naturally ventilated enclosure are presented in figure 4 , where the buoyancy driven flow through the vents is determined by the integral relation (2.13). The depth of the ambient layer 

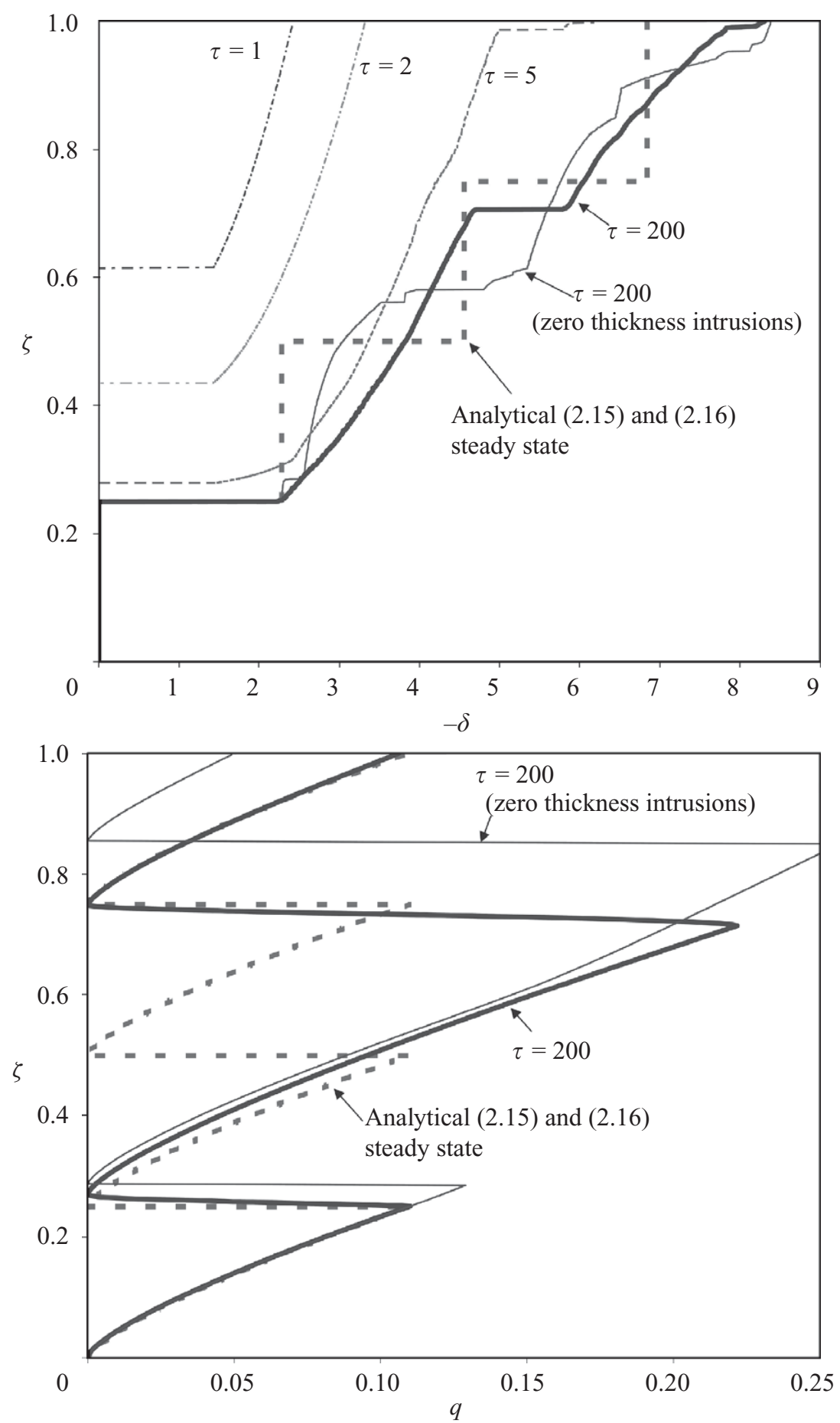

FiguRE 3. Comparison of the predicted ambient stratification $\delta$ and plume flow rate $q$ in a ventilated box containing a vertical distributed source with a forced ventilated flow rate of $q_{v e n t}=0.1097$ : (dashed line) following the model postulated by Linden et al. (1990); (fine line) instantaneous result for $\tau=200$ using the present numerical analysis with infinitesimally thin intrusions; (bold line) steady-state result for numerical simulation assuming intrusions of finite thickness with relative thickness, $\delta_{\text {int }}=0.02$ and $\tau=200$.

increases with increasing non-dimensional vent area, $A^{*} /(\alpha H L)$, as expected. The relationship between $q_{v e n t}$ and $A^{*} /(\alpha H L)$ predicted by our theory is shown in figure 5 and may be approximated by $q_{v e n t}=0.64\left(A^{*} /(\alpha H L)\right)^{0.62}$.

Our experiments described in $\S 3$ were based on the saline solution technique used by many other researchers. Our vertical distributed source of buoyancy was a permeable 

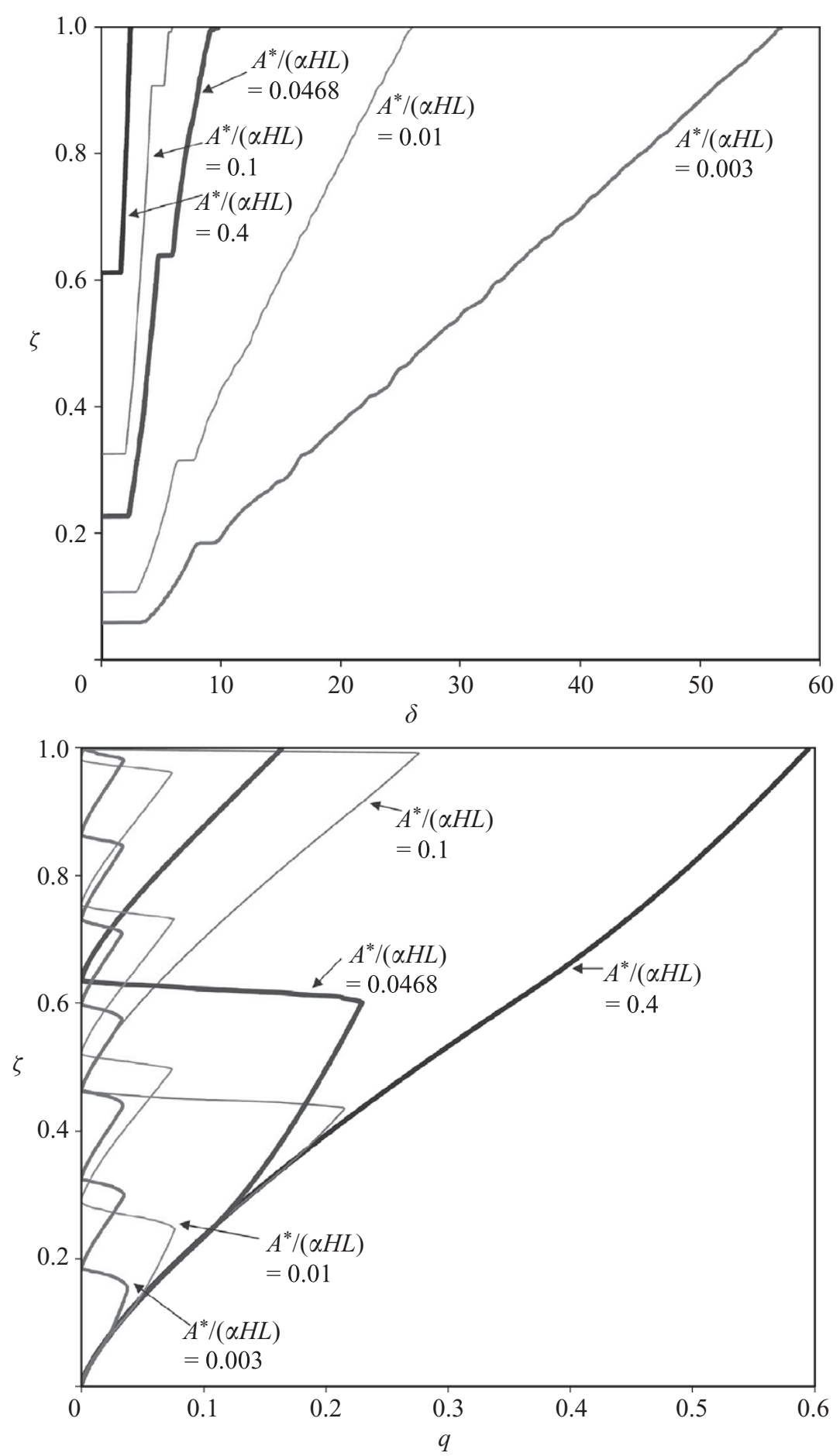

FIGURE 4. Ambient stratification $\delta$ and plume flow $q$ in a naturally ventilated box containing a vertical distributed source with a range of non-dimensional vent areas, $A^{*} /(\alpha H L)$, for $\tau=50$.

membrane fed by a chamber holding concentrated salt solution. This arrangement had the advantages that all other walls of the enclosure were effectively 'adiabatic' and a much larger Grashof number was achievable than if a thermal experiment of the same scale had been used. In addition, the flow could be readily visualized. However, this type of source meant firstly that a finite mass flux entered the ambient from the source and secondly that the buoyancy flux per unit area was not uniform with respect to height since: (i) the hydrostatic pressure across the membrane varied with height and (ii) the local buoyancy flux was dependent on the local difference in 


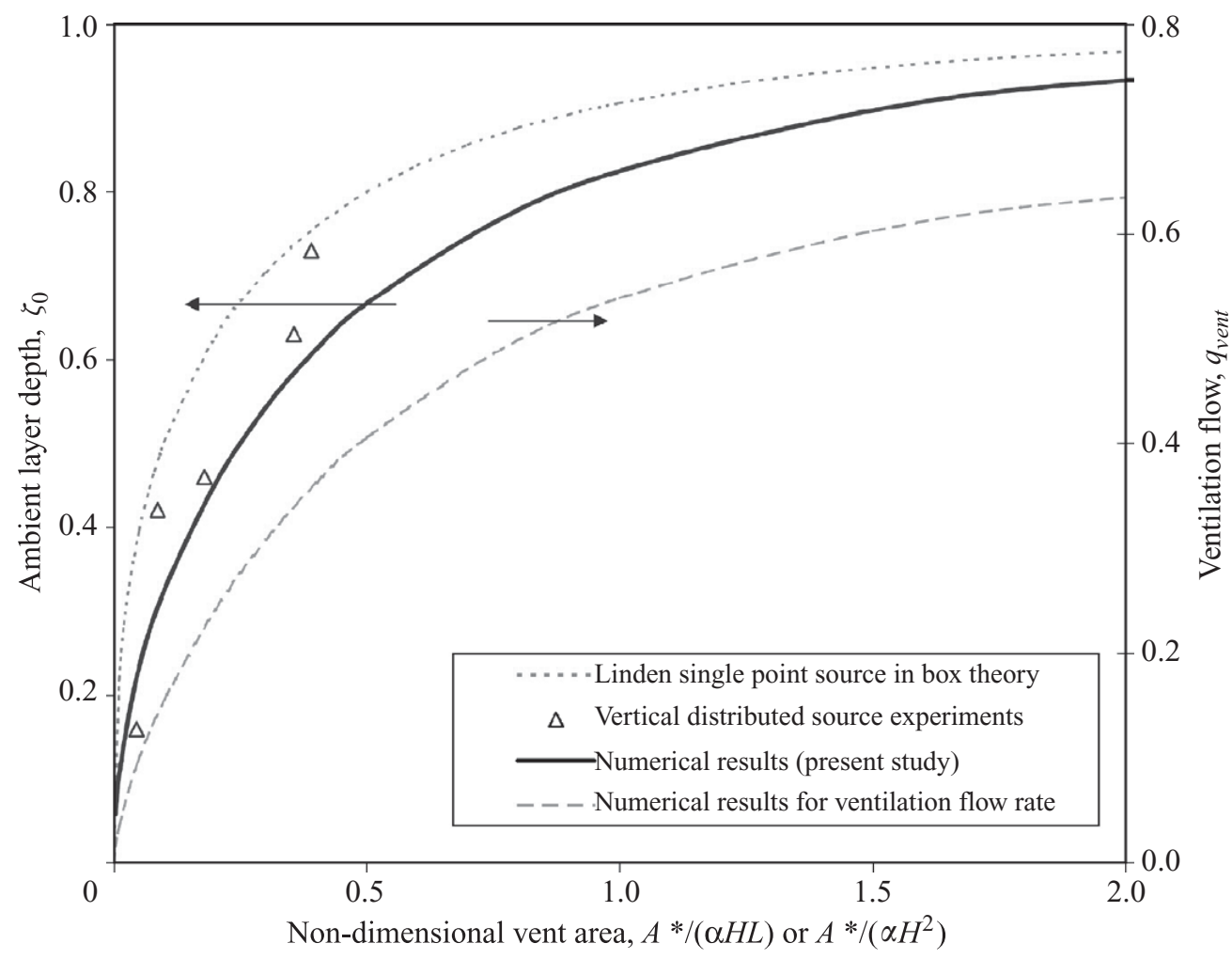

FIGURE 5. Naturally ventilated filling box. Comparison of analytical models and measurements for steady-state conditions: (dotted line) analytical prediction by Linden et al. (1990) of the ambient layer depth $\zeta_{0}$ as a function of non-dimensional vent area for a single-point source of buoyancy on the base of the box; (solid line) numerical prediction from the present study of the ambient layer depth for a vertically distributed source; (symbols) experimental results for a vertically distributed source; (dashed line) variation of the steady-state ventilation flow rate $q_{v e n t}$ as a function of non-dimensional vent area for a vertically distributed source.

buoyancy of the source and ambient fluids either side of the membrane. Our numerical model was therefore enhanced to model this practical situation by determining the mass flux through the membrane, and hence buoyancy flux, as a function of height knowing the permeability of the membrane.

\section{Experiments}

In order to validate the model presented in $\S 2$ we chose the well established saline solution technique (e.g. Linden et al. 1990), rather than thermal experiments, so as to minimize the complications arising from flow phenomena within the enclosure that were extraneous to the problem at hand. In particular, the saline solution technique avoids the difficulty of non-adiabatic walls, which, in thermal experiments, leads to boundary layers or plumes forming on surfaces other than the primary vertical, distributed source of buoyancy. Moreover, the saline solution technique facilitated relatively clear visualization of the transient flows and stratification within the enclosure could be measured by determining the spatial variation of electrical conductivity of the ambient fluid. The significant challenge of realizing a distributed source of buoyancy on one vertical wall of the enclosure using the saline solution technique was met by employing a sintered polytetrafluoroethylene (PTFE) plate, approximately $0.5 \mathrm{~cm}$ thick with a pore size of order $40 \mu \mathrm{m}$, which was supplied by saline solution under low pressure from a header tank. A schematic of the equipment configured for a naturally ventilated experiment is shown superimposed 


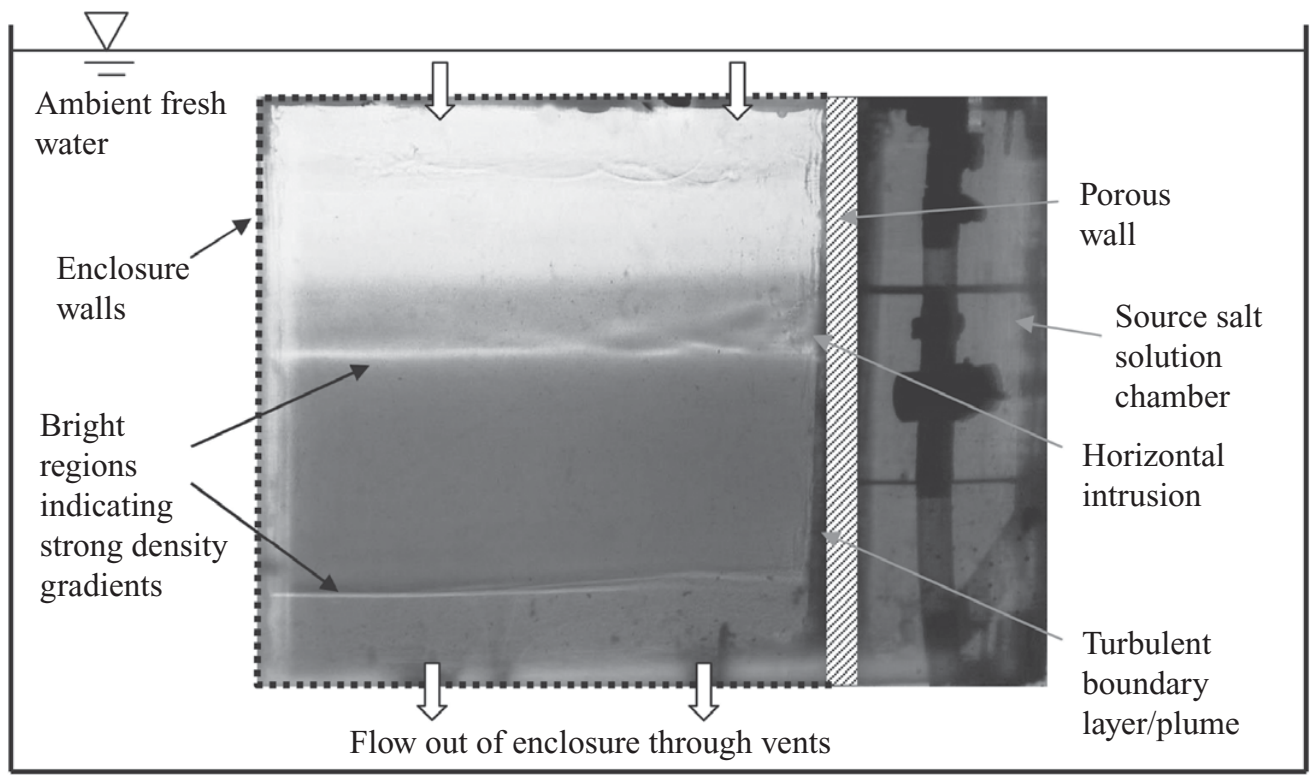

FIGURE 6. Annotated digitized image showing a typical naturally ventilated experiment at $t=30 \mathrm{~min}$. Dye was added to the source salt solution to aid in the interpretation of the density field. The porous wall on the right provided a vertically distributed plane source of buoyancy. Experimental conditions $A^{*}=0.56 \mathrm{~cm}^{2}, \Phi=1.5 \mathrm{~cm}^{2} \mathrm{~s}^{-3}$.

on a shadowgraph of a typical experiment in figure 6 . The pore size of the porous wall was selected so as to provide an appropriate balance between the need for a low pressure drop across the plate (to avoid distortion and leakage problems) whilst maintaining a sufficiently uniform flow rate through the plate with respect to height. Nevertheless, it was not possible in practice to generate a uniform buoyancy flux over the whole surface of the source using the porous wall technique, and it should be noted that significant non-uniformity in the volume and buoyancy fluxes through the plate arose naturally from the increase in hydrostatic pressure across the plate with increasing depth and from the difference in density between the source fluid and ambient fluid on opposite sides of the porous plate.

\subsection{Determination of entrainment constant for a vertically distributed plane source}

A series of experiments was conducted in a sealed acrylic enclosure of internal dimensions $25 \times 25 \times 25 \mathrm{~cm}$ to determine whether the entrainment assumption was valid for the plume developing adjacent to the distributed source and to estimate the value of the entrainment constant if appropriate. The enclosure was vented through its upper boundary to allow displacement of fluid due to the entry of a finite volume of salt solution through the porous wall. A thin acrylic plate was held between the porous plate and the interior of the enclosure prior to the start of an experiment. At the start of each experiment, $t=0 \mathrm{~s}$, the gate was carefully raised by hand, resulting in the development of a plume adjacent to the porous plate almost instantaneously. The development of the stratification in the enclosure was visualized using a shadowgraph and the location of the first front was highlighted by adding a food dye to the source fluid. Each experiment was recorded using a CCD camera, and digitized images were generated via a computer and frame grabber image capture system. Processing of the digitized images was carried out using the image-analysis system DigiFlow (Dalziel 1993) to provide quantitative information on the location of the first front. The position of the first front was determined by processing the video recording of each experiment in such a way that a representative vertical column of pixels within the 


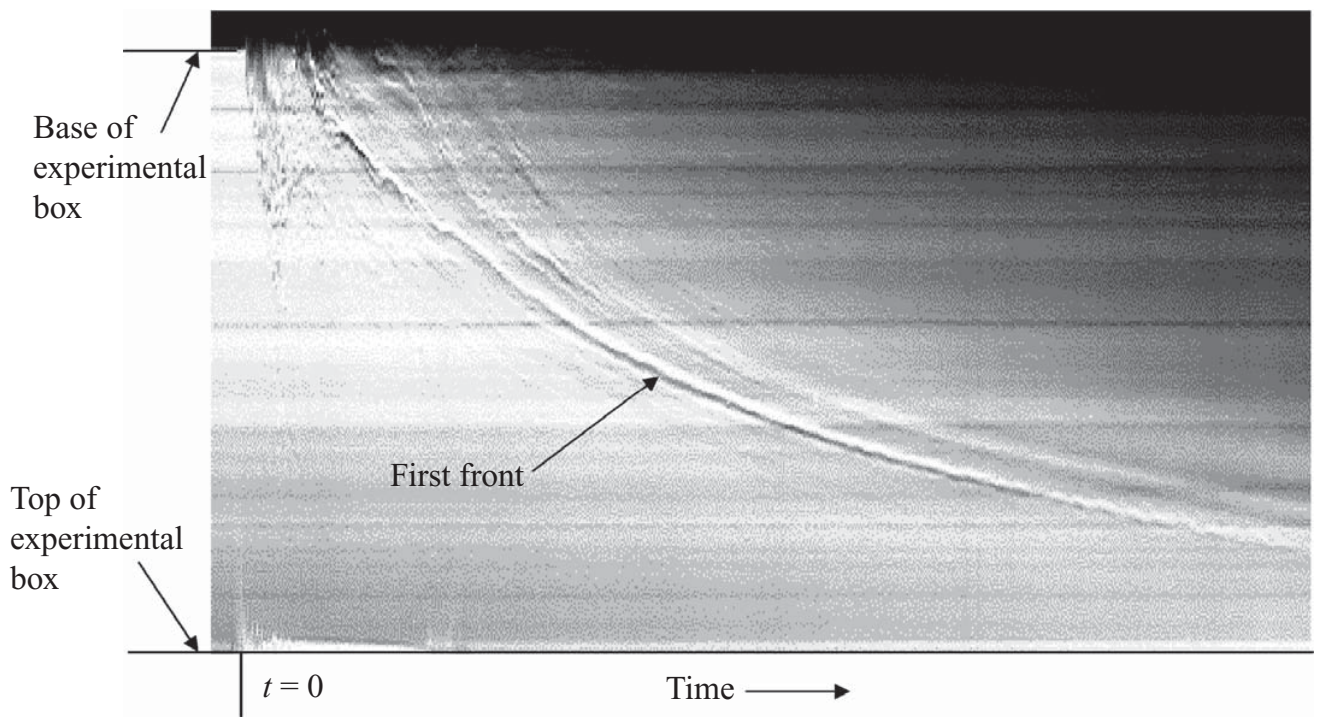

FiguRE 7. An example of a post-processed, digitized image of an experiment to track the elevation of the first front in a sealed box containing a plane vertical distributed source of buoyancy. Moving from left to right, each column of pixels in the image above represents intensities along a single vertical line of the intensity in the pre-processed image at that time. (The experiments were carried out with a vertical source of negative buoyancy. The image here has been inverted to match the representation in $\S 1$ ).

enclosure was extracted from a series of successive images and assembled to provide a composite image similar to that shown in figure 7 . In this way the position of the first front could be determined accurately as a function of time.

A comparison of the first front height as a function of non-dimensional time $\tau$ for six experiments together with that predicted by (2.10) is shown in figure 8 . There was some variation between experiments in the establishment of the initial buoyant layer as a result of: (i) difficulties in starting the plume consistently and (ii) the initial transient mixing due to the plume reaching the floor of the box and the subsequent gravity current development. Thus, an appropriate offset in $\tau$ was made for each experiment.

An entrainment constant of $\alpha=0.02$ was found to give the best least squares fit of these first front elevation data to (2.10) and it can be seen that the entrainment assumption is approximately valid for non-dimensional time $0<\tau<2.5$. For $\zeta<0.2$ the entrainment assumption did not appear to hold, most probably because the boundary layer for small $\zeta$ was laminar and thus entrainment in this region was very substantially less than that in an idealized turbulent plume.

It should be noted that (2.10) is strictly only valid for an ideal source with fully developed turbulent plume flow over the entire source and with zero mass flux and uniform buoyancy flux with respect to height at the source, whereas our experimental method required a non-zero mass flux at the source. Moreover, in practice this mass flux was not uniform with respect to height due to the variation of pressure drop across the source membrane resulting from the different density profiles in the source fluid and fluid in the enclosure. This in turn led to a non-uniform buoyancy flux through the membrane with height. To determine how these non-zero and non-uniform mass and buoyancy fluxes affected the entrainment coefficient deduced from experiments we modified our numerical code to model the non-uniform source characteristics. Using the measured pressure drop coefficient of the source membrane, the known buoyancy of the source fluid and the hydrostatic pressure applied at the top of 


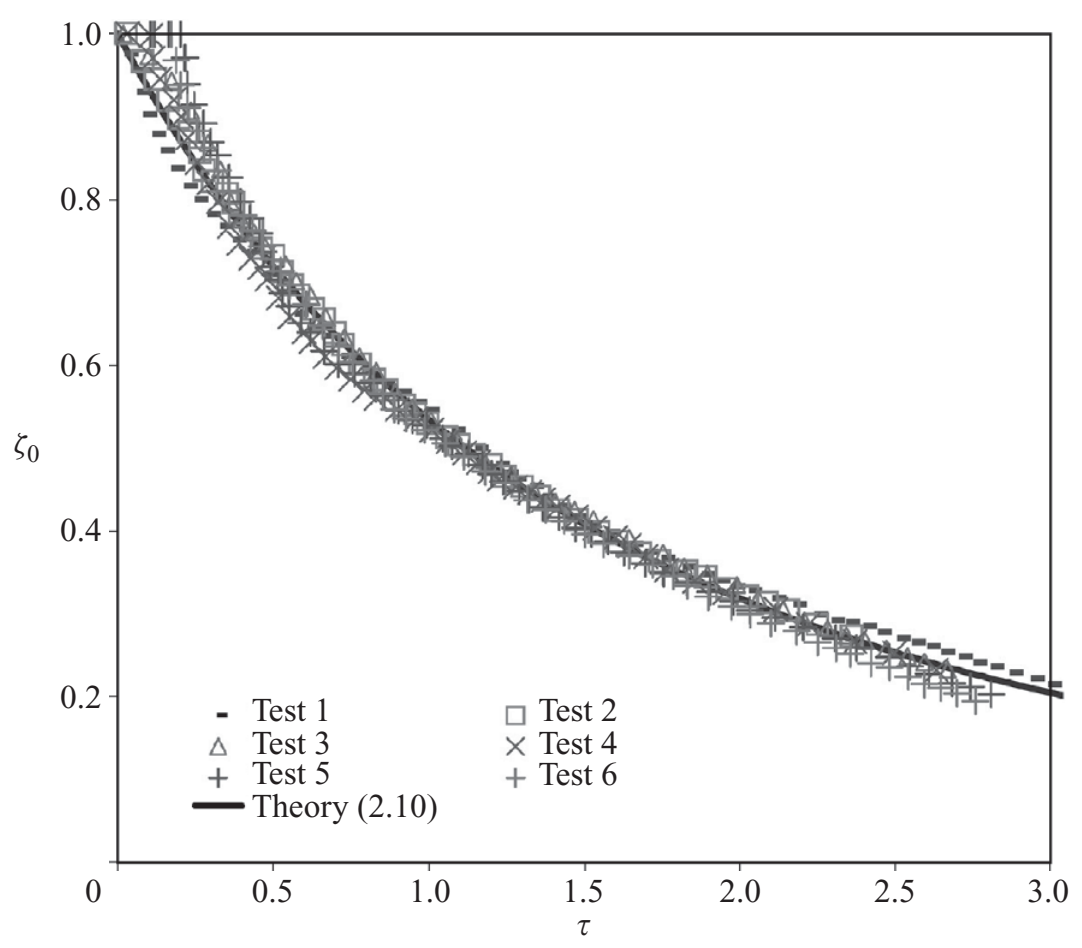

FIgURE 8. Position of the first front as a function of non-dimensional time in a sealed enclosure containing a plane vertically distributed source of buoyancy where non-dimensional time $\tau$ was determined assuming an entrainment constant $\alpha=0.02$. Tests 1,2 and 3: source velocity $v_{0}=0.05 \mathrm{~cm} \mathrm{~s}^{-1}, \Phi=0.56 \mathrm{~cm}^{2} \mathrm{~s}^{-3}$; Tests 4,5 and 6 : source velocity $v_{0}=0.10 \mathrm{~cm} \mathrm{~s}^{-1}$, $\Phi=1.10 \mathrm{~cm}^{2} \mathrm{~s}^{-3}$. (Note that the start times of the experiments have been offset to take account of the different times required for initial development of the plume following manual raising of the gate adjacent to the buoyancy source at $\tau=0$ ).

membrane for each experiment the mass and buoyancy fluxes from the source could be calculated as a function of height and time. Two examples of the comparison between this numerical prediction of the height of the first front (employing non-ideal source characteristics) and experiment are shown in figure 9. It was found that when the non-zero and non-uniform mass flux through the source was modelled in the Germeles code, and the upstream portion of the plume was assumed to be laminar with negligible entrainment, then a value of $\alpha \approx 0.03$ provided the best correlation of the six experiments reported here. This value of entrainment constant is significantly lower than that previously reported for either a plane plume above a horizontal line source of buoyancy or a wall plume immediately above a horizontal line source, which is of order 0.08 (e.g. Bloomfield \& Kerr 1998; Baines 2002). Thus, it is acknowledged that there is a degree of uncertainty in the magnitude of our estimated entrainment coefficient given the limitations of the present experimental apparatus.

\subsection{Stratification in a box with forced ventilation}

The effect of a steady forced displacement ventilation flow on the stratification within the enclosure was then determined using a second acrylic tank measuring $41.2 \mathrm{~cm}$ in height, $15.0 \mathrm{~cm}$ in width and $60.0 \mathrm{~cm}$ in length where the buoyancy source formed one of the $15 \mathrm{~cm}$ wide walls. The stratification in the ambient fluid was determined by means of an electrical conductivity probe which was mounted on a computer-controlled traverse and connected to a commercial conductivity meter. The construction of the probe was similar to that described by Leppinen (1997). The forced ventilation flow was effected by introducing a flow of fresh water through a 


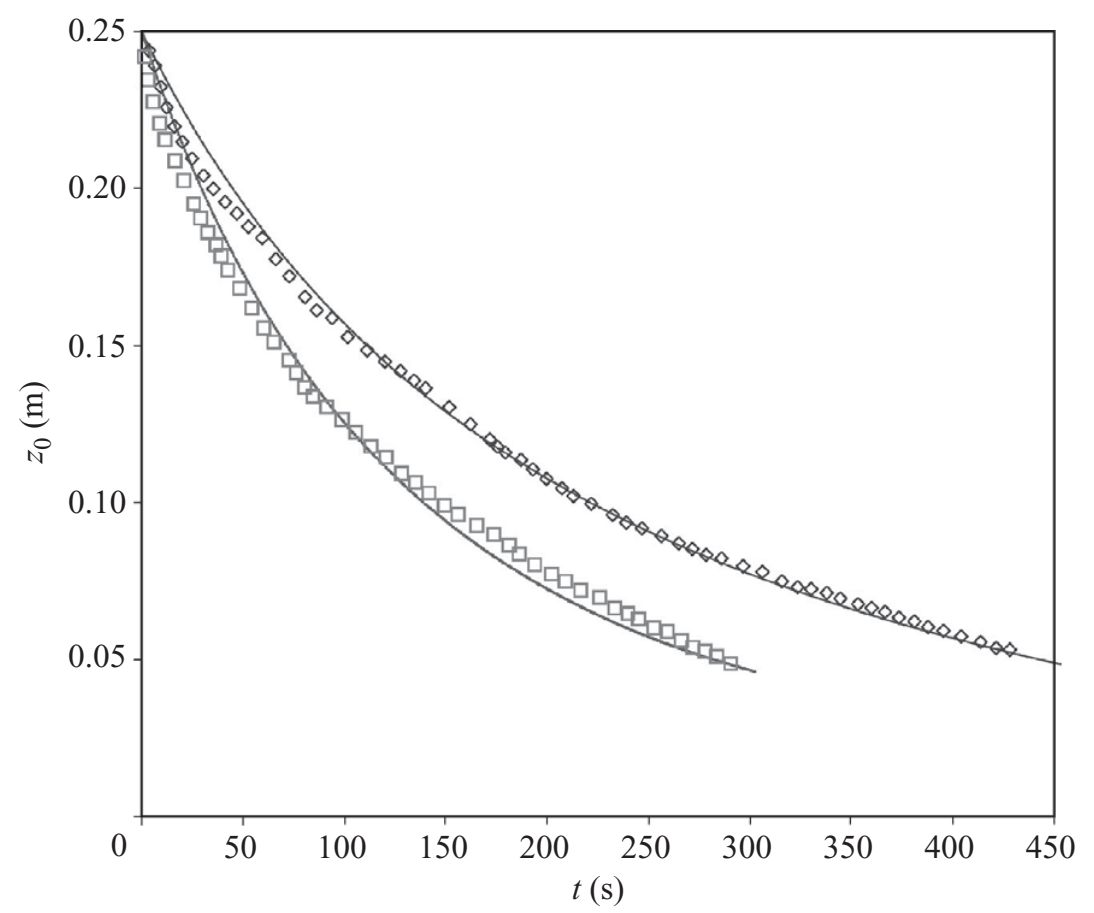

Figure 9. Position of the first front as a function of time in a sealed enclosure containing a plane, vertically distributed source of buoyancy. Experiments: squares - test 6; diamonds test 1: and best fit numerical results $(\alpha=0.03)$ where the buoyancy source was modelled as having non-zero mass and buoyancy fluxes that both varied as a function of height due to changes in applied hydrostatic pressure across the porous source plate with height, and with the upstream portion of the plume assumed laminar with negligible entrainment.

second porous wall across the top of the tank, the effect of which was to eliminate any inlet high velocities that would otherwise disturb the stable stratification in the box had the ambient fluid been introduced through pipes or vents.

\subsection{Transient development of stratification in the ventilated enclosure}

Development of the stratification in the ambient fluid followed that predicted by the theory of $\S 2$ qualitatively. Results from a typical transient experiment are shown in figure 10. It can be seen that there was no distinct step change in density evident across the first front of the descending buoyant layer. This was due to the mixing observed to occur when the starting plume reached the bottom of the enclosure at the start of each experiment. Although there was qualitative agreement between the experiments and the stratification predicted from the numerical model detailed in $\S 2.5$, the experiments were somewhat variable due in part to the fact that the flow through the porous wall was very sensitive to the condition of the membrane which was difficult to maintain. In addition, for many of the experiments it was necessary to maintain the flow rate of source fluid through the membrane at a value that was comparable to or greater than the ventilation flow rate of buoyant fluid exiting the enclosure. Nevertheless the theoretical model appears to give a reasonably quantitative representation of the stratification found in the ambient fluid in practice given the uncertainties associated with the experiments, as shown for the two examples in figure 11.

A number of significant difficulties were faced when attempting to represent the idealized flow depicted schematically in figure 1 at the laboratory scale, and these difficulties resulted in differences in detail between the predicted and experimentally observed density fields. Firstly, the upstream portion of any natural convection boundary layer arising from a real vertical distributed buoyancy source will be 

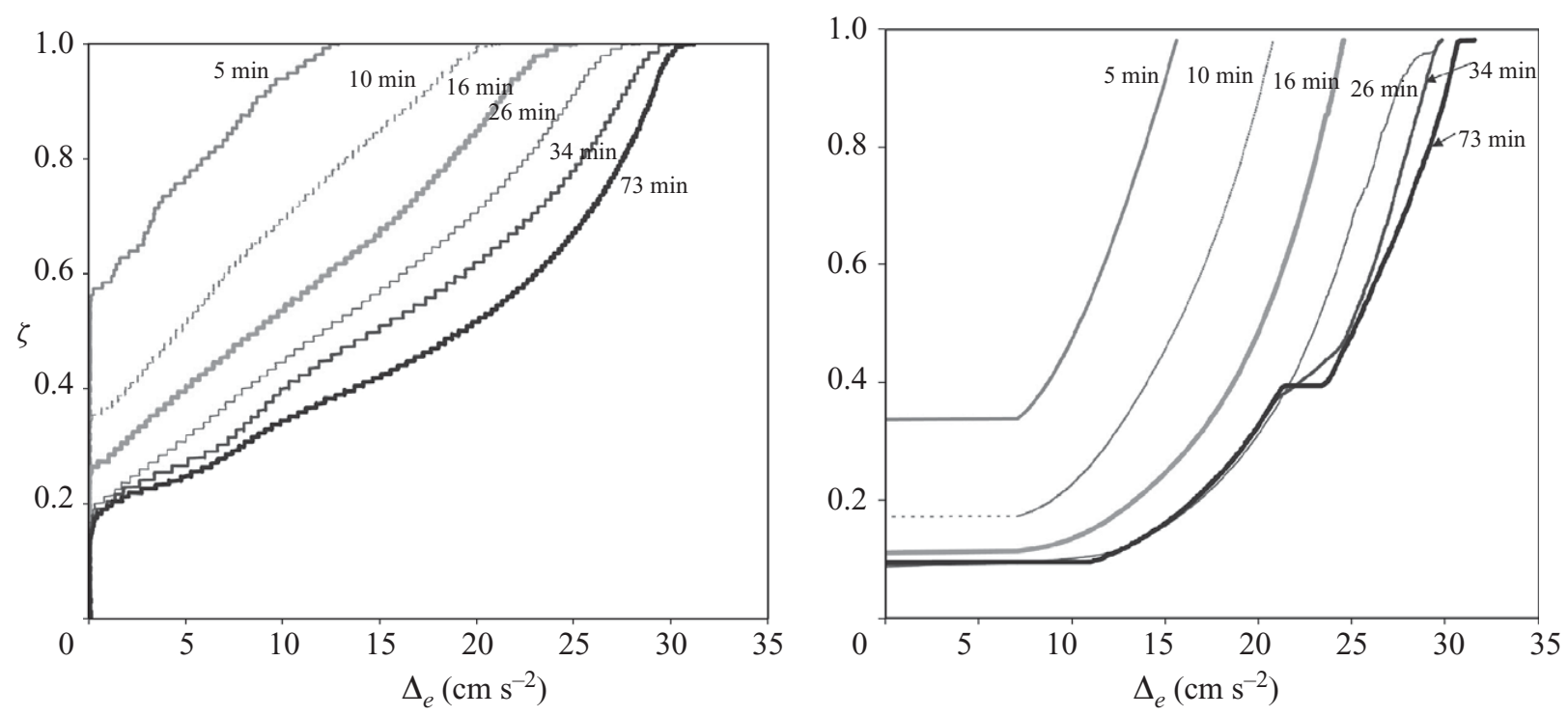

FiguRE 10. (a) Development of stratification in a typical forced ventilation experiment plotted as ambient fluid buoyancy $\Delta_{e}$ as a function of height and of time in minutes from the start of the experiment (total forced ventilation flow rate $Q_{\text {vent }} L=36.7 \mathrm{~cm}^{3} \mathrm{~s}^{-1}$; source flow rate $Q_{0}=32.1 \mathrm{~cm}^{3} \mathrm{~s}^{-1}$; source density $\rho_{0}=1.038 \mathrm{~g} \mathrm{~cm}^{-3}$ ). (b) Numerical prediction of the stratification for the same experimental conditions.

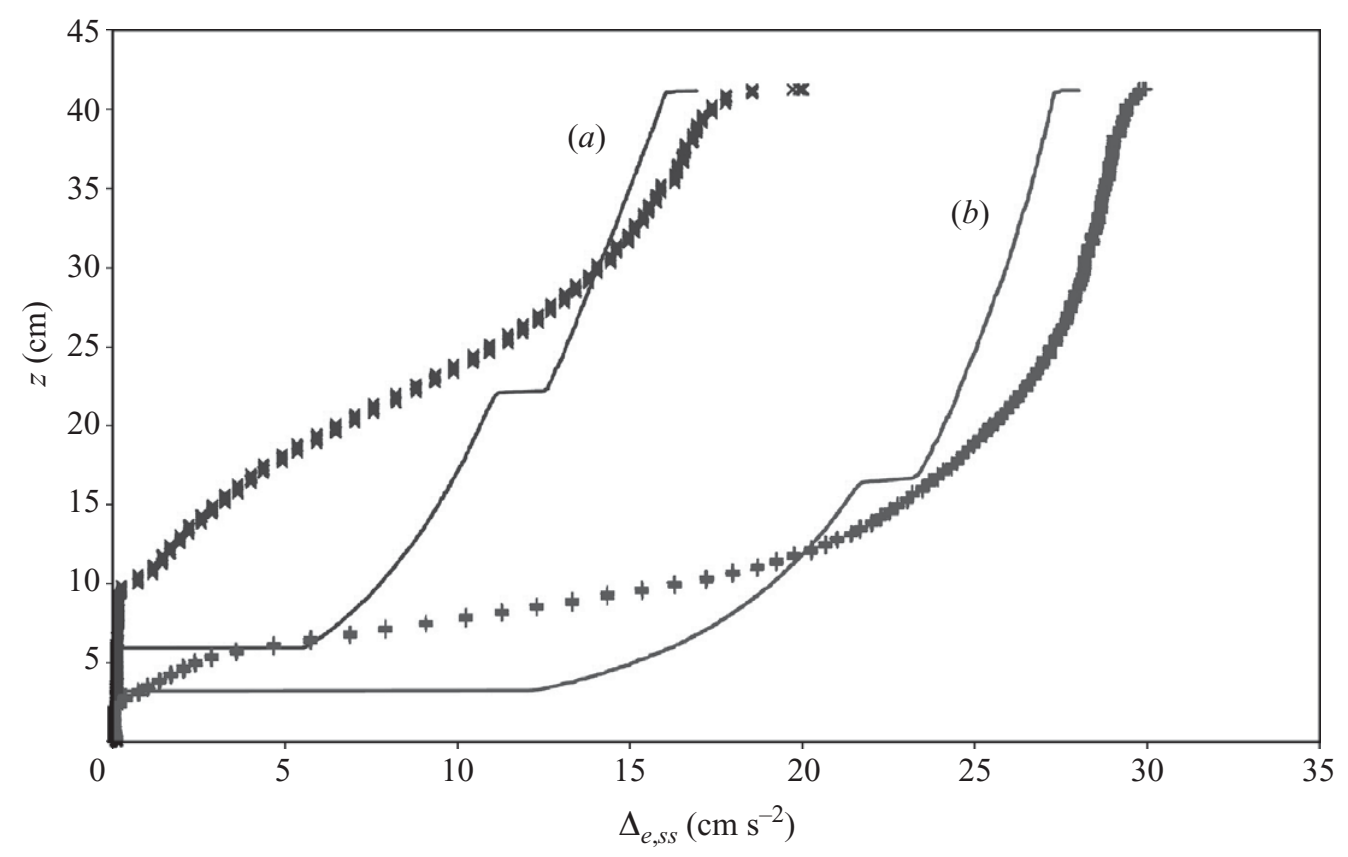

FIGURE 11. Comparison of experimental results (symbols) and numerical model (continuous lines) for two typical steady-state forced ventilation experiments. Approximate flow conditions: (a) total forced ventilation flow rate $Q_{\text {vent }} L=28.8 \mathrm{~cm}^{3} \mathrm{~s}^{-1}$; source flow rate $Q_{0}=22.5 \mathrm{~cm}^{3} \mathrm{~s}^{-1}$; source density $\rho_{0}=1.023 \mathrm{~g} \mathrm{~cm}^{-3} ;(b)$ total forced ventilation flow rate $Q_{\text {vent }} L=41.7 \mathrm{~cm}^{3} \mathrm{~s}^{-1}$; source flow rate $Q_{0}=33.7 \mathrm{~cm}^{3} \mathrm{~s}^{-1}$; source density $\rho_{0}=1.031 \mathrm{~g} \mathrm{~cm}^{-3}$.

laminar and in our experiments this region occupied up to a quarter of the total height of the source. Secondly, the experiments did not reveal the presence of a sharp first front at $\zeta_{0}$, which is due to the tendency of the initial buoyant layer laid down by the plume to overturn as discussed by Baines \& Turner (1969) and Kaye \& Hunt (2007). It is also clear that our relatively simple model of detrainment, whereby the entire plume is assumed to leave the vertical wall to form an intrusion, 
does not account for a number of secondary flow mechanisms. These include the fact that the density profile across the plume at a given elevation is not uniform and thus buoyant elements within the plume will reach neutral buoyancy conditions over a range of heights within the enclosure. It was also observed experimentally that some fluid immediately adjacent to the vertically distributed source (including the laminar sublayer) continued to descend beyond the level of the intrusion. Moreover, the interaction of the plume with sharp changes in the density of the ambient with respect to height were not modelled, e.g. in respect of mixing and generation of waves and instabilities. For example, the entrainment of a buoyant layer by an impinging plume (Kumagai 1984) was not included in our present analysis, however, this could be added in a similar way to Cooper \& Linden (1996).

\section{Conclusions}

We have examined the stratification that develops in an enclosure containing a vertically distributed source of buoyancy. Both the unventilated 'filling box' case, in which the enclosure is completely sealed, and the ventilated 'filling box' case, in which openings at floor and ceiling level connect the enclosure to an exterior quiescent body of fluid of uniform density, are considered. The latter case allows a steady-state flow field and stratification to be established for either the forced or naturally ventilated configurations.

The uniform buoyancy flux from the vertically distributed source of buoyancy drives a turbulent boundary layer and a new theoretical model of the resulting stratification within the enclosure has been developed by the present authors. This is based on the assumption that the boundary layer adjacent to the buoyancy source is self-similar and plume-like, and, thus, the entrainment assumption of Morton et al. (1956) may be invoked. The system of governing differential equations has been solved using a numerical technique similar to that of Germeles (1975). The theoretical predictions have been validated by comparison with laboratory experiments in a water tank using saline solutions (injected through a vertical porous wall of the box) as buoyancy inputs.

For the ventilated filling box, a vertically distributed plane buoyancy source results in a stable and complex stratification. In contrast to the case of a plume developing above a point or horizontal line source of buoyancy, a plume developing adjacent to a vertically distributed source of buoyancy in a ventilated enclosure may attain a buoyancy less than the local stratified ambient at a given height. In such a case the plume will detrain and form a horizontal intrusion.

Linden et al. (1990) postulated that a steady-state stratification made up of a finite number of distinct, fully mixed layers, each of the same depth, will develop. However, we believe that this type of stratification cannot be realized in practice since it is theoretically unstable with respect to small perturbations in the ambient density field if the detraining plume is assumed to be comprised of fluid of a single value of buoyancy (as was the case in the analysis of Linden et al. 1990). Our numerical analysis has demonstrated that if intrusions resulting from the detrainment of a plume are assumed to have a finite range of buoyancy (resulting from the fact that plumes in practice have non-uniform buoyancy profiles) then a steady-state stratification within the enclosure is achieved.

The numerical and experimental results were in qualitative agreement and the entrainment coefficient for the plume adjacent to the vertical, distributed plane source was found to be in the range $0.02 \leqslant \alpha \leqslant 0.03$ (assuming top-hat profiles within the 
plume). However, the authors acknowledge that there remains a degree of uncertainty in the quantitative value of this constant, particularly since the buoyancy source in the saline solution experiments necessarily resulted in the delivery of non-uniform volume and buoyancy fluxes with respect to height. Moreover, the small scale of the experiments precluded an exact comparison with the theory because of the existence of a laminar section of the plume adjacent to the vertically distributed source and because the turbulence generated within the rather small enclosures led to smoothing of some of the sharp interfaces. It is hoped that future thermal experiments with a buoyancy source of uniform heat flux will provide a more definitive validation of the theory presented here.

The authors would like to acknowledge the support of the Engineering and Physical Sciences Research Council in providing financial support for G. R. Hunt and a Visiting Research Fellowship for P. Cooper in support of some of this work. We thank three anonymous referees for their valuable commentary on our first draft of this paper. We would also like to acknowledge the work of our research students in providing assistance in regard to the experiments, notably Cameron Hough and Ole-Jørgen Myrtrøen.

\section{REFERENCES}

Baines, P. G. 2002 Two dimensional plumes in stratified environments. J. Fluid Mech. 471, 315-337.

BAINES, W. D. \& TuRner, J. S. 1969 Turbulent buoyant convection from a source in a confined region. J. Fluid Mech. 37, 51-80.

Bloomfield, L. J. \& KerR, R. C. 1998 Turbulent fountains in a stratified fluid. J. Fluid Mech. 358, $335-356$.

Bolster, D. \& CAUlfield, C. P. 2008 Transients in natural ventilation - a time-periodically varying source. Build. Serv. Engng Res. Technol. 29, 119-135.

Chen, Z. D., Li, Y. \& Mahoney, J. 2001 Natural ventilation in an enclosure induced by a heat source distributed uniformly over a vertical wall. Build. Environ. 36, 493-501.

Cooper, P. \& Linden, P. F. 1996 Natural ventilation of an enclosure containing two buoyancy sources. J. Fluid Mech. 311, 153-176.

Dalziel, S. B. 1993 Rayleigh-Taylor instability: experiments with image analysis. Dyn. Atmos. Oceans 20, 127-153.

Germeles, A. E. 1975 Forced plumes and mixing of liquids in tanks. J. Fluid Mech. 71, 601-623.

Kaye, N. B. \& Hunt, G. R. 2007 Overturning in a filling box. J. Fluid Mech. 576, 297-323.

KumagaI, M. 1984 Turbulent buoyant convection from a source in a confined two-layered region. J. Fluid Mech. 147, 105-131.

LePpinen, D. M. 1997 Aspects of convection. PhD thesis, DAMTP, University of Cambridge.

Linden, P. F. 1999 The fluid mechanics of natural ventilation. Annu. Rev. Fluid Mech. 31, 201-238.

Linden, P. F. \& COOPER, P. 1996 Multiple sources of buoyancy in a naturally ventilated enclosure. J. Fluid Mech. 311, 177-192.

Linden, P. F., Lane-SerfF, G. F. \& Smeed, D. A. 1990 Emptying filling boxes, the fluid mechanics of natural ventilation. J. Fluid Mech. 212, 309-335.

McDougall, T. J. 1978 Bubble plumes in stratified environments. J. Fluid Mech. 85, 655-672.

Morton, B. R., TAYlor, G. I. \& Turner, J. S. 1956 Turbulent gravitational convection from maintained and instantaneous sources. Proc. R. Soc. Lond. A 234, 1-23.

Sandberg, M. \& Lindstrom, S. 1987 A model for ventilation by displacement. In Proceedings of Roomvent 87: First Intl Conf. on Air Distribution in Rooms, Stockholm, Sweden.

Vliet, G. C. \& LiU, C. K. 1969 An experimental study of turbulent natural convection boundary layers. ASME J. Heat Transfer 91, 517-531.

Wells, M. G., Griffiths, R. W. \& Turner, J. S. 1999 Competition between distributed and localized buoyancy fluxes in a confined volume. J. Fluid Mech. 391, 319-366.

Worster, M. G. \& Huppert, H. E. 1983 Time-dependent density profiles in a filling box. J. Fluid Mech. 132, 457-466. 